\title{
MAGNETIC SUSCEPTIBILITY OF PbSnMnTe IN THE TRANSITION REGION BETWEEN FERROMAGNETIC AND SPIN GLASS PHASE**
}

\author{
T. Story, R.R. Galązka \\ Institute of Physics, Polish Academy of Sciences \\ Al. Lotników 32/46, 02-668 Warszawa, Poland \\ P.J.T. Eggenkamp, H.J.M. Swagten, and W.J.M. DE Jonge \\ Department of Physics, Eindhoven University of Technology \\ P.O. Box 513, $5600 \mathrm{MB}$ Eindhoven, The Netherlands
}

The ac magnetic susceptibility $(\chi)$ was measured in the temperature range $\dot{T}=(1.5 \div 50) \mathrm{K}$ for $\mathrm{Pb}_{1-x-y} \mathrm{Sn}_{y} \mathrm{Mn}_{x}$ Te samples with a composition $y=0.72, x=0.015 \div 0.04$ and carrier concentrations in the range $p=$ $(2.85 \div 14) \times 10^{20} \mathrm{~cm}^{-3}$. The breakdown of ferromagnetism and the formation of a spin glass phase are observed with an increasing carrier concentration. It is experimentally evidenced as continuous deviation of $\chi(T)$ from the characteristic critical behavior observed for ferromagnetic samples.

PACS numbers: 75.50.Pp, 75.50.Lk

The concentration of carriers is the basic parameter determining the ma.gnetic properties of IV-VI semimagnetic semiconductors (diluted magnetic semiconductors). It was demonstrated [1] that $\mathrm{Pb}_{1-x-y} \mathrm{Sn}_{y} \mathrm{Mn}_{x}$ Te samples with the carrier concentration exceeding the threshold value $p_{\mathrm{t}} \cong 3 \times 10^{20} \mathrm{~cm}^{-3}$ are ferromagnetic at liquid helium temperatures. The microscopic mechanism responsible for the formation of the ferromagnetic phase is the Ruderman-Kittel-Kasuya-Yosida (RKKY) indirect interspin exchange interaction via free carriers [2, 3]. It was recently discovered [4] that this ferromagnetic order can be destroyed by the further increase of the carrier concentration beyond $p_{\mathrm{t}} \cong 10^{21} \mathrm{~cm}^{-3}$. In the same time both the strength of the interaction and the magnitude of the magnetic moment of manganese ions (determined from high temperaturc magnetic measurements) are practically unchanged. The interpretation of this effect is based on the oscillatory character of the RKKY interaction. The spin glass phase is expected to be observed whenever the mean distance between spins $\left(R \propto x^{-1 / 3}\right)$ is larger than the distance at which the RKKY exchange integral starts to oscillate $\left(R_{0} \propto p^{-1 / 3}\right)$,

*This work is supported in part by the Committee for Scientific Research under grant 204829101 . 
thereby introducing the competition of ferro- and antiferromagnetic interactions. When $R<R_{0}$ the spectrum of possible exchange interactions is dominated by ferromagnetic couplings and, consequently, the formation of the ferromagnetic phase is expected. The line separating these two regimes is determined by the condition $R=R_{0}$, i.e. $(p / x)=$ const. The aim of this communication is to study the process of the transformation of the ferromagnetic phase to the spin glass one as a function of carrier concentration.

We performed the ac magnetic susceptibility measurements in the temperature range $T=(1.6 \div 50) \mathrm{K}$. Based on the high temperature data we determine the paramagnetic Curie temperature $\theta-$ the parameter which reflects the total strength of the exchange couplings between spins. The temperature at which the ferromagnetic phase transition takes place (the ferromagnetic Curie temperature $T_{\mathrm{C}}$ ) is determined as the point of the maximum slope of the $\chi(T)$ plot. Carrier concentration is determined by standard de IIall effect measurements. The investigated samples cover the composition range $y=0.72, x=0.015 \div 0.04$ and carrier concentration range $p=(2.85 \div 14) \times 10^{20} \mathrm{~cm}^{-3}$.

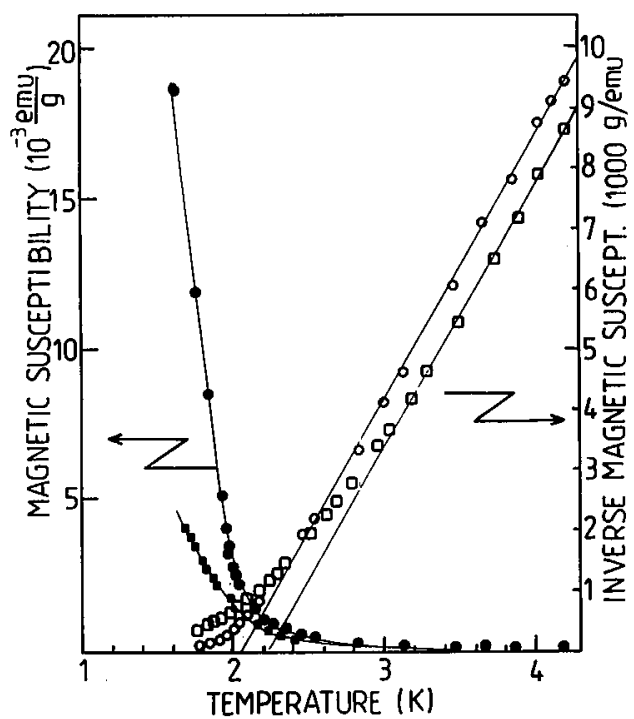

Fig.1



Fig. 2

Fig. 1. The temperature dependence of the magnetic susceptibility of two samples of $\mathrm{Pb}_{0.265} \mathrm{Sn}_{0.72} \mathrm{Mn}_{0.015} \mathrm{Te}$ : the ferromagnetic sample with the carrier concentration $p=$ $5.8 \times 10^{20} \mathrm{~cm}^{-3}$ (circles) and the spin glass like sample with $p=1.25 \times 10^{21} \mathrm{~cm}^{-3}$ (squares). The right hand scale is for the plot of temperature dependence of the inverse magnetic susceptibility (open symbols).

Fig. 2 The temperature dependence of the magnetic susceptibility of four samples of $\mathrm{Pb}_{0.26} \mathrm{Sn}_{0.72} \mathrm{Mn}_{0.02} \mathrm{Te}$ with different carrier concentrations given in the figure in units $10^{20} \mathrm{~cm}^{-3}$. 


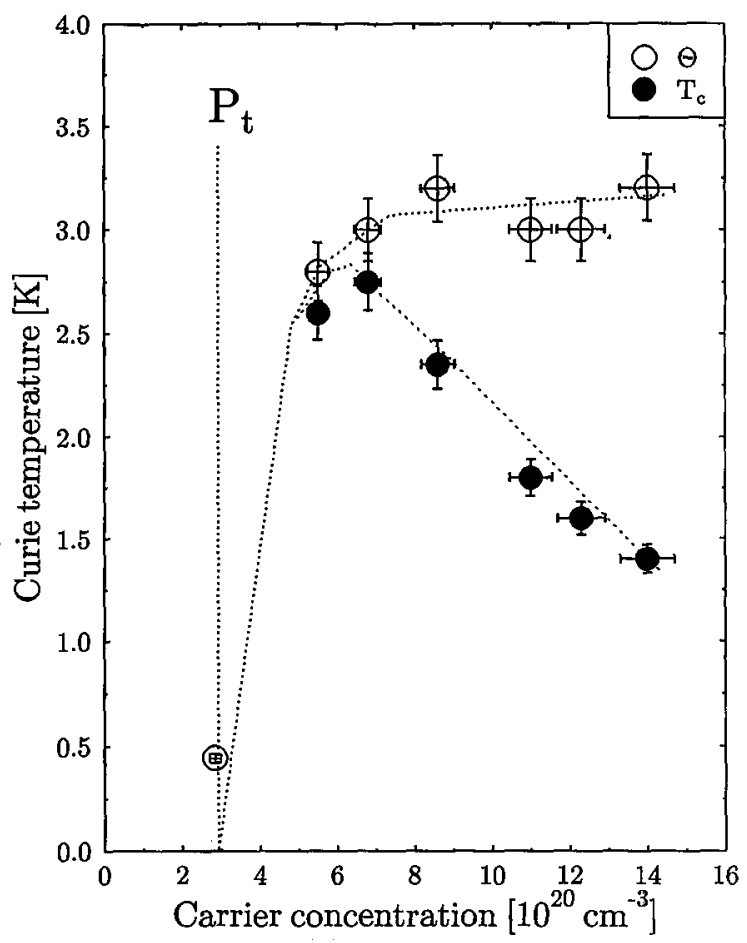

Fig. 3. The carrier concentration dependence of the paramagnetic (open circles) and the ferromagnetic (full circles) Curie temperature for $\mathrm{Pb}_{0.26} \mathrm{Sn}_{0.72} \mathrm{Mn}_{0.02} \mathrm{Te}$.

The typical tempcrature dependence of the magnetic susceptibility of $\mathrm{PbSnMnTe}$ samples is presented in Fig. 1. The sample with carrier concentration $p=5.8 \times 10^{20} \mathrm{~cm}^{-3}$ exhibits the ferromagnetic phase transition at $T_{\mathrm{C}} \cong 1.9$ $\mathrm{K}$ slightly below the paramagnetic Curie temperature $\theta \cong 2.15 \mathrm{~K}$. The sample with the carrier concentration $p=1.25 \times 10^{21} \mathrm{~cm}^{-3}$ does not exhibit any magnetic phase transition till $T=1.6 \mathrm{~K}$, despite the fact that the RKKY exchange interaction is in this case stronger (as evidenced by the larger value of $\theta$ ). Similar effect is observed for the samples containing 2 at.\% of Mn (see Fig. 2) but seems to be absent in the samples containing 4 at.\% of Mn. It agrees well with the predictions of the simple model discussed above. The low temperature magnetic susceptibility of four samples of $\mathrm{Pb}_{0.26} \mathrm{Sn}_{0.72} \mathrm{Mn}_{0.02} \mathrm{Te}$ with different carrier concentrations is presented in Fig. 2. One can notice a continuous suppression of the Curie temperature of the ferromagnetic phase transition and the onset of the formation of a spin glass like phase. The more extensive experimental studies of SnMnTe samples with the carrier concentration $p \cong 2 \times 10^{21} \mathrm{~cm}^{-3}$ [4] (including the measurements of specific heat and frequency dependence of magnetic properties) strongly suggest that, ultimately, a spin glass phase is observed in these crystals. The continuous character of this process is also demonstrated in Fig. 3 where both Curie temperatures are ploted versus carrier concentration. One can notice that a con- 
tinuous increase in $\theta$ is accompanied by a decrease of the actual temperature of magnetic phase transition.

In conclusion, we observed experimentally a continuous process of the breakdown of the ferromagnetic order in $\mathrm{PbSnMnTe}$, being replaced by a spin glass like phase. The magnetic phase observed in the samples from the transition region cannot be described neither as ferromagnetic nor as canonical spin glass. These experimental findings confirm the interpretation of this effect based on the oscillatory character of the RKKY interaction.

\section{References}

[1] T. Story, R.R. Galązka, R.B. Frankel, P.A. Wolff, Phys. Rev. Lett. 56, 777 (1986).

[2] H.J.M. Swagten, W.J.M. de Jonge, R.R. Gałazka, P. Warmenbol, J.T. Devreese, Phys. Rev. B 37, 9907 (1988).

[3] T. Story, G. Karczewski, L. Świerkowski, R.R. Galazzka, Phys. Rev. B 42, 10477 (1990).

[4] W.J.M. de Jonge, T. Story, H.J.M. Swagten, P.J.T. Eggenkamp, Europhys. Lell. $17,631(1992)$. 